\title{
Editorial
}

\section{Nanomaterials from Natural Products for Industrial Applications}

\author{
Sohel Rana, ${ }^{1}$ Raul Fangueiro, ${ }^{1}$ Vijay K. Thakur, ${ }^{2}$ Mangala Joshi, ${ }^{3}$ \\ Sabu Thomas, ${ }^{4}$ and Bodo Fiedler ${ }^{5}$ \\ ${ }^{1}$ Centre for Textile Science and Technology (2C2T), University of Minho, Guimarães, Portugal \\ ${ }^{2}$ Enhanced Composites \& Structures Centre, Cranfield University, Cranfield, UK \\ ${ }^{3}$ Department of Textile Technology, Indian Institute of Technology, Delhi, India \\ ${ }^{4}$ School of Chemical Science, Mahatma Gandhi University, Kottayam, Kerala, India \\ ${ }^{5}$ Institute of Polymer and Composite, Technical University of Hamburg, Hamburg, Germany
}

Correspondence should be addressed to Sohel Rana; soheliitd2005@gmail.com

Received 22 November 2017; Accepted 23 November 2017; Published 26 December 2017

Copyright ( 92017 Sohel Rana et al. This is an open access article distributed under the Creative Commons Attribution License, which permits unrestricted use, distribution, and reproduction in any medium, provided the original work is properly cited.

This special issue is focused on the nanomaterials derived from natural products and their application in different industrial sectors.

Nanotechnology has emerged as one of the most promising tools for creating high performance and multifunctional materials for various industrial applications. Looking at the growing concern about the environment and sustainability, nanomaterials from natural sources are receiving great attention in the scientific community as well as in industrial sectors. A wide variety of nanomaterials synthesized from natural products possess exceptional strength characteristics, light weight, transparency, and excellent biocompatibility and are, therefore, promising materials for coatings, packaging, medicine, construction, electronics, filtration, transportation, and other areas. Among various techniques, electrospinning has been considered to be a simple and versatile technique for producing continuous nanofibres from natural polymers. Therefore, besides particles, it has been possible to produce continuous fibres and textiles based on these nanomaterials.

Within the range of natural nanomaterials, nanocellulose has attracted a special attention and has been widely used for industrial applications. Nanocellulose has been either synthesized through bottom-up approach using bacteria (known as bacterial cellulose) or produced from plant cellulose through physical (such as ultrasonication, homogenization, and ball milling) or chemical methods (such as acid hydrolysis) or their combination in the form of nano-fibrillated cellulose (NFC) and nano-crystalline cellulose (NCC). Nanocellulose possesses high mechanical properties (NCC's axial Young's modulus: 120-220 GPa, transverse modulus: 11-57 GPa, tensile strength: $7.5 \mathrm{GPa}$ ), high surface area, and interesting barrier and optical properties and is, therefore, considered to be an excellent reinforcement for composite materials. This special issue presented a number of sources for production of nano cellulose and its application in different areas. For example, M. Hassanzadeh et al. produced NFC from two underutilized Appalachian hardwoods, Northern red oak (Quercus rubra) and yellow poplar (Liriodendron tulipifera) and explored the possibility of using the produced NFC as templates for antimicrobial copper for packaging and medical/pharmaceutical applications. J. Li et al. produced NFC from poplar and cotton sources and used for the reinforcement of Polylactic Acid (PLA) composites. An interesting application of copper immobilized NFC has been reported by $\mathrm{C}$. W. Owens et al. as catalyst for the synthesis of 1-benzyl-4-phenyl-1H-1,2,3-triazole from benzyl azide and phenylacetylene with a yield of over $90 \%$.

A number of attempts have been made to control the size and morphology as well as functionality of produced nanocellulose targeting different applications. These approaches either include pretreatment of cellulosic materials prior to nanocellulose extraction or functionalization of produced nanocellulose using different chemical or physical 
methods. In this special issue, Y. Chen et al. presented a green pretreatment approach with exoglucanase and endoglucanase enzymes to produce NFC with controlled length for special applications.

Nanomaterials produced by bacteria are also getting tremendous attention in recent times. Bacterial cellulose is an appropriate example of this type of materials with high purity, crystallinity, and excellent mechanical properties. Minicells generated by lactic acid bacteria are another type of nanosized particles with huge application potential in drug delivery systems for treatment of cancer, microbial infection, and other diseases, as discussed in the review paper by $\mathrm{H}$. N. Nguyen et al. included in the present special issue.

Nanoclays such as montmorillonite (MMT), kaolinite, and saponite are other natural nanomaterials which have been widely used for reinforcement of polymeric as well as cementitious composites. Reinforcement of polymers with nanoclays improves their physical, mechanical, and gas barrier properties. On the other hand, in case of cementitious materials, nanoclays are used to improve the mechanical performance, resistance to chloride penetration, and selfcompacting performance and to reduce the permeability and shrinkage properties. Nanoclays find diversified applications in cosmetics, drug delivery systems, pollution control and water treatment, food industry, and so on. An application of nanoclays to improve the mechanical and barrier performance of whey protein isolate nanocomposites films for food packaging applications has been presented in this special issue by $\mathrm{K}$. Müller et al. The use of other inorganic nanomaterials such as MCM-41 silicate and Y-Zeolites, deposited with a bioactive plant extract for medical applications, has been discussed by M. A. Hernández et al. Some other nanomaterials from natural products for medical applications, discussed in this issue, include nanoemulsion produced from the extract of Amazonian andiroba plant oil as reported by S. S. R. Milhomem-Paixão et al. and nanocomposite films of hyaluronic acid containing metal nanoparticles as discussed by G. Cárdenas-Triviño et al.

However, despite promising properties and applications, further research and developments are extremely essential for nanomaterials obtained from various natural products. Production of these nanomaterials at industrial scale needs costeffective production processes, improved production rate, and consistent quality as well as predictable and consistent performance of nanomaterials based products. This special issue presented some of the recent research approaches to study and optimize the properties of a few natural product based nanomaterials and highlighted their enormous application potential in diversified industrial sectors.

Sohel Rana

Raul Fangueiro

Vijay K. Thakur

Mangala Joshi

Sabu Thomas

Bodo Fiedler 

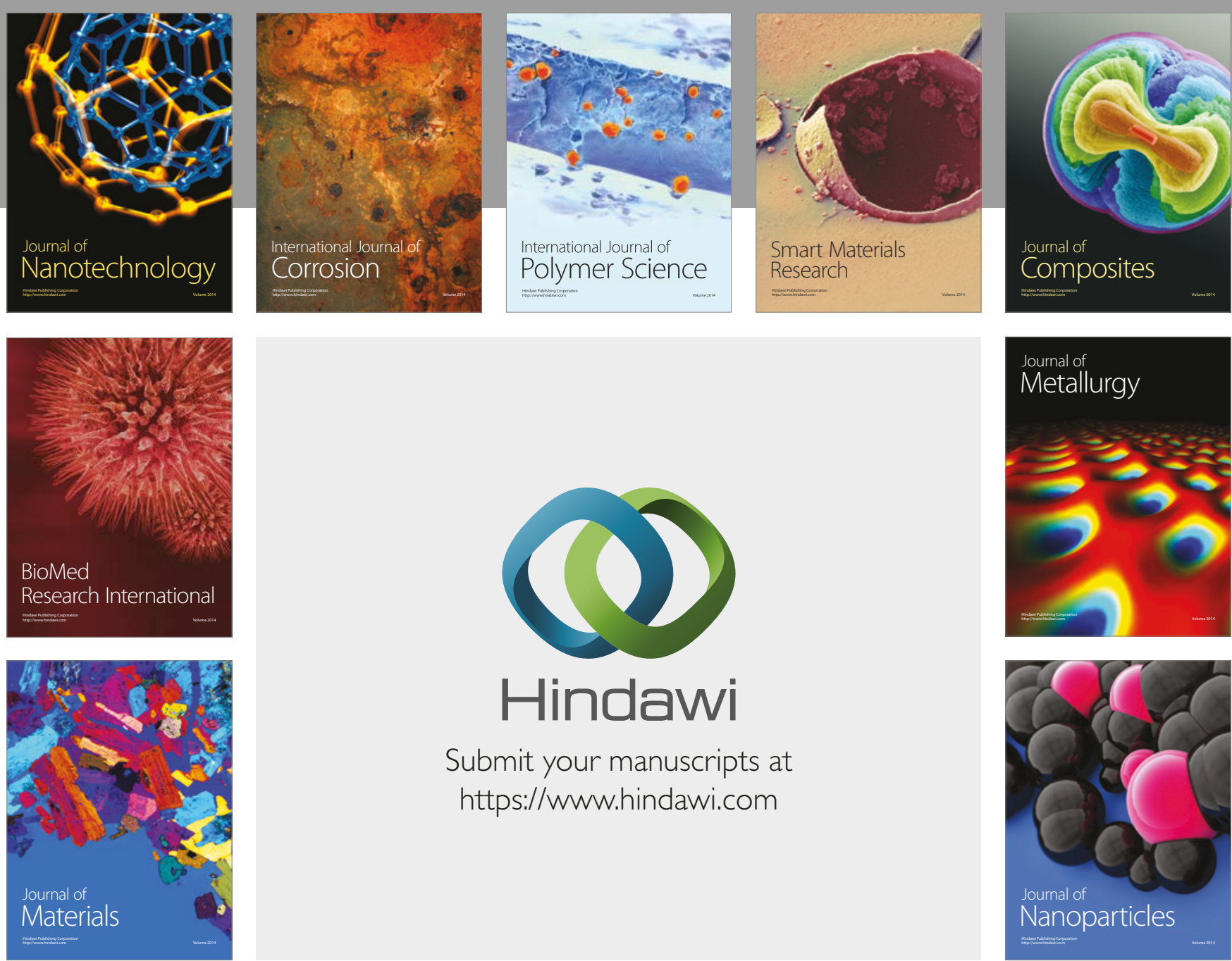

\section{Hindawi}

Submit your manuscripts at

https://www.hindawi.com
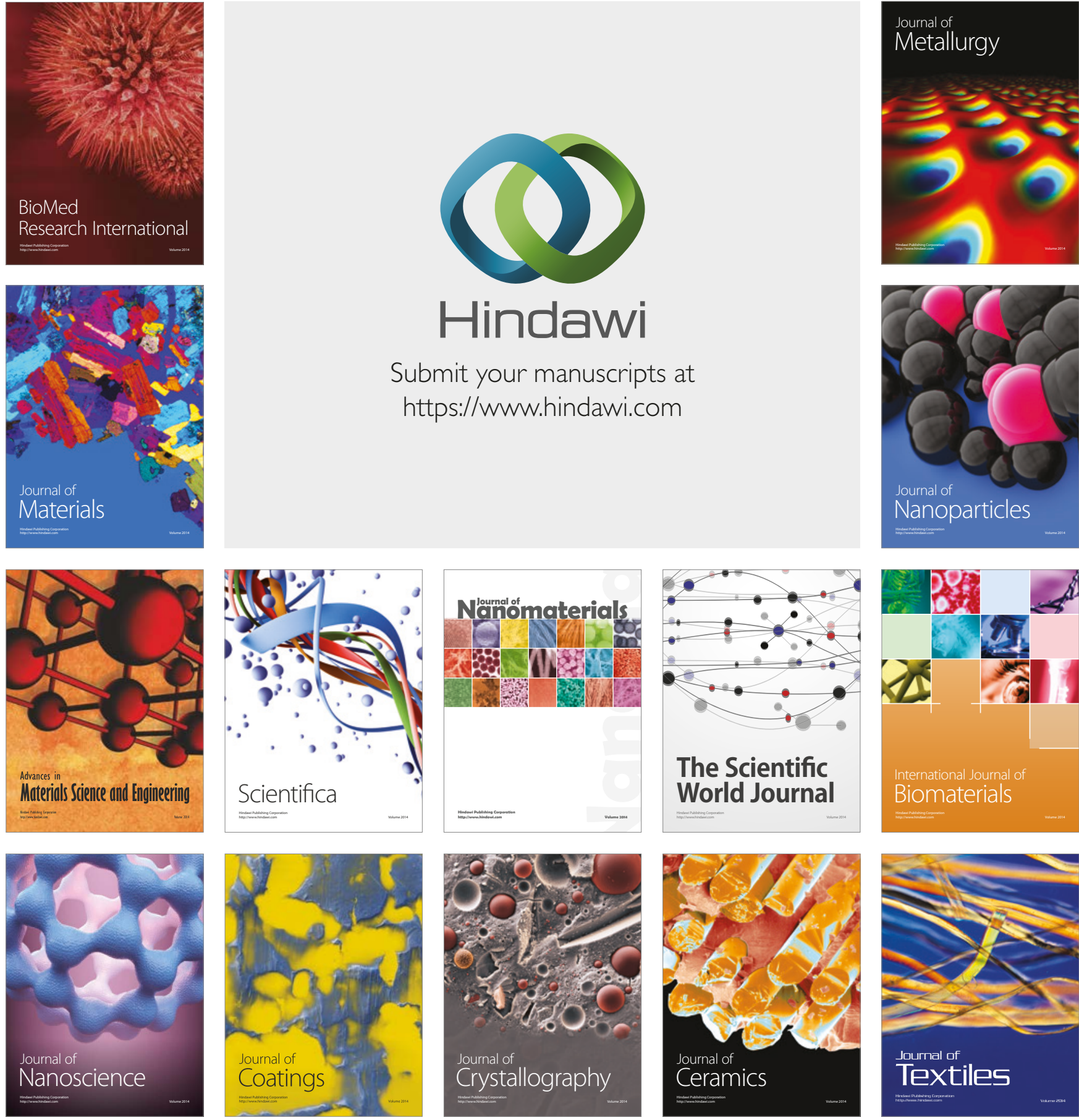

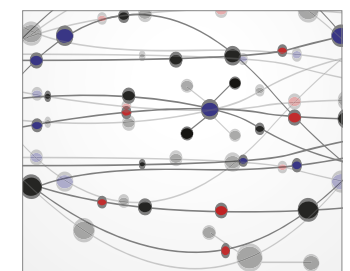

The Scientific World Journal
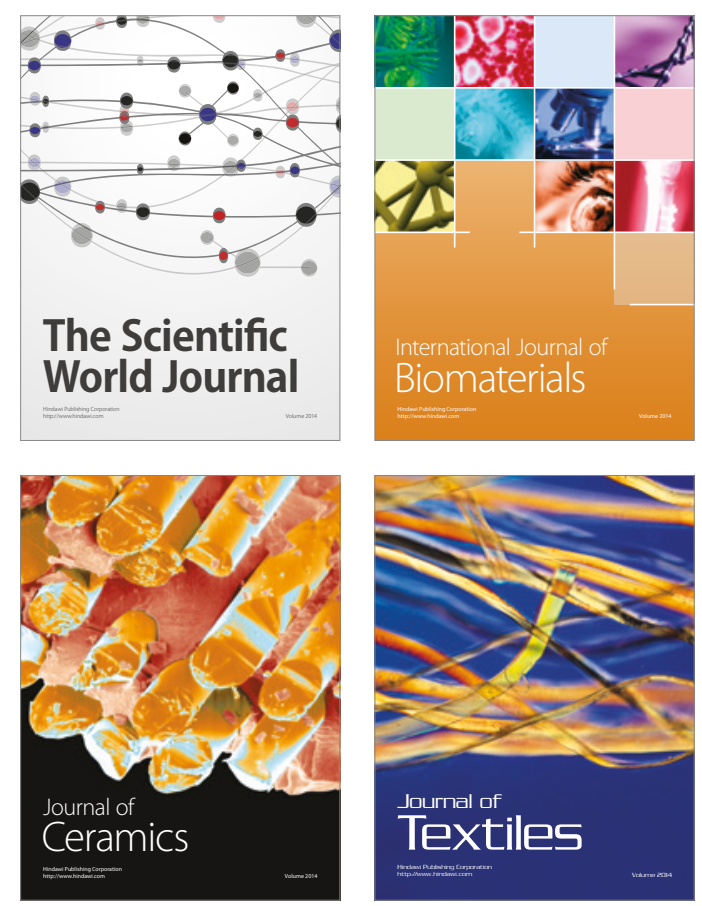